\title{
The hormonal Zeitgeber melatonin: role as a circadian modulator in memory processing
}

\author{
Oliver Rawashdeh* and Erik Maronde \\ Dr. Senckenbergische Anatomie III, Institute of Cellular and Molecular Anatomy, Goethe-University, Frankfurt, Hessen, Germany
}

\section{Edited by:}

Kristin Eckel-Mahan, University of

California at Irvine, USA

\section{Reviewed by:}

Urs Albrecht, University of Fribourg, Switzerland

Miou Zhou, University of California, Los Angeles, USA

*Correspondence:

Oliver Rawashdeh

Dr. Senckenbergische Anatomie III, Institute of Cellular and Molecular

Anatomy, Goethe-University,

Theodor-Stern-Kai 7, Hs 26, D-60590

Frankfurt, Hessen, Germany.

e-mail: rawashdeh@em.uni-

frankfurt.de
The neuroendocrine substance melatonin is a hormone synthesized rhythmically by the pineal gland under the influence of the circadian system and alternating light/dark cycles. Melatonin has been shown to have broad applications, and consequently becoming a molecule of great controversy. Undoubtedly, however, melatonin plays an important role as a time cue for the endogenous circadian system. This review focuses on melatonin as a regulator in the circadian modulation of memory processing. Memory processes (acquisition, consolidation, and retrieval) are modulated by the circadian system. However, the mechanism by which the biological clock is rhythmically influencing cognitive processes remains unknown. We also discuss, how the circadian system by generating cycling melatonin levels can implant information about daytime into memory processing, depicted as day and nighttime differences in acquisition, memory consolidation and/or retrieval.

Keywords: circadian rhythms, learning, melatonin, zebrafish, memory, pineal, hormone

\section{THE CIRCADIAN SYSTEM}

We are living within a cycling environment in which changes occur on a daily basis with a period length of $24 \mathrm{~h}$ and others that alternate with a periodicity ranging from several months (seasonal), to annual cycles. Importantly, not only are our surroundings rhythmic, but humans, as well as the simplest of organisms display endogenous rhythms. Nevertheless, much of today's identified rhythmic physiological processes are not coincidentally in or out of phase with exogenous rhythms but are in part driven and reset by cycling physical cues.

The field of biological research that focuses on endogenous rhythms is known today as "circadian biology." The term "circadian" was first coined by Franz Halberg in the late 1950s derived from latin "circa" meaning "about" and "dies" known as "day", thus, referring to endogenous rhythms that cycle with a period length of about a day. Rhythms with significantly shorter or longer periodicities than $24 \mathrm{~h}$ are known as ultradian and infradian rhythms, respectively.

Environmental rhythms as in the cycling of daylight, resulting from the earth's rotation about its axis, and to which temperature rhythms are coupled to, have an impact on our endogenous clock. Permission of such an influence requires specialized sensory structures to detect daily environmental fluctuations. Eyes for example, sense daily rhythms in light/dark cycles, thus, transmitting lighting information via the retinohypothalamic tract, in humans and other mammalian species, to a central circadian clock located in the suprachiasmatic nucleus (SCN). The SCN in turn communicates information about time chemically, by regulating rhythmic melatonin synthesis for instance, to other downstream oscillators within the hierarchal structure of the circadian system. We, therefore, can say that daily exogenous rhythms function as Zeitgebers (German for time cues or time-givers) for our internal circadian system. But, what are they good for? Zeitgebers are important for the daily resetting of the biological clock. Meaning, an overt rhythm, as in locomotor activity, maintains in synchrony (of particular phase relationship) with its environment. This is important because the periodicity of biological clocks generally deviate from cycling with a period of exactly $24 \mathrm{~h}$. Therefore, biological rhythms with periods $>$ or $<$ than $24 \mathrm{~h}$, need to be reset backwards or forwards, respectively, on a daily basis.

Melatonin synthesis, locomotor activity (Cahill et al., 1998; Hurd et al., 1998), feeding behavior (Stokkan et al., 2001), core body temperature (Castillo et al., 2005) and memory processing (Chaudhury and Colwell, 2002; Fernandez et al., 2003; Lyons et al., 2005) are just a few examples of either direct circadian outputs or indirectly modulated outputs of the circadian system. This review will focus on the role of the hormonal time cue, melatonin, on the circadian modulation of memory processing.

\section{MELATONIN A HORMONE OF THE PINEAL GLAND}

Melatonin ( $N$-acetyl-5-methoxytryptamine) is a hormone first discovered in the late 1950s by Aaron Lerner (Lerner et al., 1958). Melatonin in most vertebrates has been shown to be rhythmic with elevated levels restricted to nighttime (Cassone, 1990) irrespective to the organism's activity profile, thus, the coined term "hormone of darkness." The functional roles of melatonin, proposed in many studies, are widespread as shown next.

\section{MELATONIN AND THE CIRCADIAN SYSTEM}

The rhythmic melatonin profile is defined mostly by the temporal regulation of arylalkyamine- $N$-acetyltransferase-mRNA at the transcriptional level (Klein et al., 1997) and the product of post-translational regulation (Pozdeyev et al., 2006). In 
mammals, the clock driving rhythmic melatonin synthesis is localized to the SCN. The SCN is coupled to the pineal gland, a photoneuroendocrine transducer, via interconnected anatomical neuronal substrates, collectively known as the photoneuroendocrine pathway (Korf et al., 1998). In contrast to SCN driven pineal melatonin synthesis, local pineal oscillator-dependent rhythmic melatonin synthesis were identified in lower nonmammalian species such as birds (Zimmerman and Menaker, 1975), sauropsids (Bartell et al., 2004), and teleosts such as zebrafish (Cahill, 1996; Kaneko et al., 2006). Interestingly, the rainbow trout (Oncorhynchus mykiss) among other salmonids, lacks a local pineal oscillator. Consequently, the shape of its rhythmic melatonin synthesis profile is entirely dependent on the subjected light/dark cycle (Kroeber et al., 2000). In addition to a circadian regulation of melatonin synthesis, light itself impacts pineal melatonin synthesis on a post-translational level (Gastel et al., 1998). Importantly, the inhibitory effect of light on melatonin synthesis seems to be preserved during evolution, irrespective of the presence of a local pineal oscillator (Gaston and Menaker, 1968; Zimmerman and Menaker, 1975; Turek et al., 1976; Takahashi and Zatz, 1982; Cassone, 1990). Evolutionary differences in the regulation of melatonin synthesis may account for differences in the importance of pineal melatonin in circadian physiology across different animal models. For example, while in sparrows and lizards, melatonin has a profound role in the circadian organization, as the stability of the circadian system is sensitive to alterations in melatonin (Binkley et al., 1971; Underwood, 1983), nocturnal rats show no measurable effect on rhythms of sleep-wake cycle and locomotor activity (Cheung and McCormack, 1982; Fisher and Sugden, 2010).

In humans, much of the focus on the role of melatonin and its therapeutic aspects is related to sleep, perhaps due to the coincidence of elevated nighttime melatonin levels, in phase with increased sleep drive and maintenance (Hughes et al., 1998; Baskett et al., 2001; Aeschbach et al., 2009). The role of melatonin on sleep onset and/or sleep maintenance is in part through the direct action of melatonin on specific brain-areas involved in sleep regulation and via its role on the circadian system (Armstrong et al., 1986; Sack et al., 1998). Lavie argued that melatonin is involved in the regulation of sleep by inhibiting centers of the central nervous system (CNS) involved in generating wakefulness (Lavie, 1997). About the same time, Sack, Lewy, and Hughes proposed an alternative hypothesis, stating that melatonin may act as a sleep promoting molecule by counteracting a signal believed to originate from the SCN to promote daily awakening (Sack et al., 1998). Nevertheless, apart from the role of melatonin on sleep, many studies propose broad applications for melatonin and its analogs on the circadian system, particularly in ameliorating the physiological and psychological effects of jet-lag (Arendt and Broadway, 1987; Petrie et al., 1989) and as a therapeutic agent for disorders related to circadian rhythm disturbances.

\section{MELATONIN AND MEMORY}

A role for melatonin in modulating complex processes such as learning and memory (Bob and Fedor-Freybergh, 2008) was first proposed when a functional impact for melatonin was discovered in brain structures such as the hippocampus known to play an important role in memory processing. At the society of neuroscience annual meeting (1995) Gonzales and Armstrong showed that melatonin interferes with neuronal transmission and long-term potentiation (LTP) in the dentate gyrus of the hippocampus. The mechanism for how melatonin may be mediating this effect was not clear. Later, Dawn Collins and Stephen Davies proposed one mechanistic pathway for melatonin action on LTP and neuronal transmission by modulating NMDA receptor functionality (Collins and Davies, 1997). The investigation by Hogan, Sherif, and Wierasszko on the inhibitory action of melatonin on LTP, concluded the effect of melatonin on LTP to be mediated by modulating synaptic efficiency and/or excitability of hippocampal neurons (Hogan et al., 2001). Rhythmicity in melatonin levels on a $\sim 24 \mathrm{~h}$ day/night cycle could thus, potentially alter hippocampal function and influence the physiology of memory processing. Later it was demonstrated that inhibition of both melatonin receptors [MT(1)/MT(2)] using antagonists such as luzindole (Dubocovich, 1988) can attenuate the effect of melatonin on excitatory postsynaptic potentials (Wang et al., 2005). This was a big leap in the field, as the approach of using melatonin receptor deficient mice proved the specificity of melatonin action on hippocampal LTP, namely, to be melatonin receptor mediated. Furthermore, by dissecting intracellular regulatory signaling pathways such as adenylyl cyclase (also known as adenylate cyclase: AC) -PKA signaling known to be modulated by melatonin (Roberson and Sweatt, 1996), Colwell's group confirmed the inhibitory action of melatonin on LTP to be linked to the action of melatonin on AC-PKA signaling in the hippocampus (Wang et al., 2005). Furthermore, the inhibitory effect of melatonin on AC-PKA pathway was shown to be mediated via the $\mathrm{MT}(2)$ melatonin receptor subtype, as the inhibitory effect on LTP was absent in both MT(2) and MT(1)/MT(2) deficient mice but not in $\mathrm{MT}(1)$ knockout animals. In addition, using a selective MT(2) receptor antagonist (4-PDOT) the inhibitory effect of melatonin on LTP could be blocked. Similar results were obtained by Reppert et al. (1995) in the SCN, showing the activation of $\mathrm{MT}(2)$ receptors by melatonin to be negatively coupled to AC and PKA activity (Reppert et al., 1995) and positively coupled to the PKC cascade (McArthur et al., 1997; Hunt et al., 2001). Larson and colleagues provided exciting results using both in vitro physiological and in vivo behavioral approaches to study the role of $\mathrm{MT}(2)$ receptors (Larson et al., 2006). Here, MT(2) deficient $\left[\mathrm{MT}(2)^{-/-}\right]$mice showed a significantly reduced LTP compared to wild type mice. Furthermore, using an in vivo learning and memory task, to evaluate spatial long-term memory (hippocampal dependent task), MT(2) ${ }^{-/}-$-mice, demonstrated impaired memory performance. The behavioral data parallels the impairment of LTP in MT $(2)^{-/-}$-mice.

Based on the here summarized data for the effect of melatonin on hippocampal neuronal electrophysiological properties, memory relevant signaling cascades and behavior (Table 1), all of which are evidence for its influence on memory processing, depicts the hypothesis, that rhythmic endogenous melatonin functions as a cycling modulator for memory processing, almost as a given. 
Table 1 | Influence of melatonin on memory performance and synaptic plasticity in diurnal and nocturnal species.

\begin{tabular}{|c|c|c|c|c|c|c|}
\hline Animal model & Activity rhythm & Learning task & Memory processes & Melatonin + LTP & LTP in Mel-R KO & Receptor mediated \\
\hline Rat & Nocturnal & & & Inhibitory & & $\mathrm{MT}(2)$ \\
\hline
\end{tabular}

Mel, melatonin; KO, knockout; MT(2), melatonin receptor 2; LTP, long-term potentiation. For references see: (Hogan et al., 2001; Wang et al., 2005; Larson et al., 2006; Rawashdeh et al., 2007).

\section{CIRCADIAN MODULATION OF MEMORY PROCESSES}

Evidence for a circadian influence on acquisition and memory processing (Gerstner et al., 2009) was first demonstrated in systems where the circadian organization was disrupted (Tapp and Holloway, 1981; Fekete et al., 1985; Antoniadis et al., 2000; Devan et al., 2001). Disturbing a system's circadian organization in rats trained for either a passive or active-avoidance learning task influences the mnemonic performance of the animal by affecting memory retrieval (Fekete et al., 1985). However, in place navigation learning, disruption of the circadian organization results in impaired memory consolidation (Devan et al., 2001). This may indicate that memory processes such as acquisition, consolidation, and retrieval for various learning paradigms may be differentially affected by the animal's circadian system. Since learning associated with diverse tasks may involve different neuronal substrates for memory processing (Gerstner et al., 2009), distinct neuroanatomical structures can be differentially regulated by the circadian system, and, thus, may influence distinctive stages of memory processing.

More recent studies, developed new methods that permit to conceive the circadian influence on memory processes while preserving the integrity of the circadian system (Chaudhury and Colwell, 2002; Fernandez et al., 2003; Lyons et al., 2005, 2006). These studies not only confirmed the involvement of the circadian system in modulating memory processes, but could also for the first time visualize the effect and confirm which memory process (acquisition, consolidation and/or retrieval) is being modulated. For example, in Aplysia californica, the formation of long-term memory (consolidation process) was shown to be most robust when animals were trained during the subjective day (the active phase) and suppressed during the subjective night (the inactive phase) (Lyons et al., 2005). In mice, two stages of memory processes (acquisition and recall) for a fear-conditioning paradigm was shown to be circadian modulated. Mice recall the conditioned task better and show an increased learning rate during their inactive phase as opposed to their active phase (Chaudhury and Colwell, 2002). Since these studies were performed under constant conditions, in the absence of external time cues, the rhythm in memory processing (acquisition, consolidation and retrieval) must be modulated by the organism's circadian system. With the current knowledge, time is ripe to address the mechanism(s) behind day/night difference in memory processing using innovative methodologies to gain new insights into how time is modulating memory.

\section{MELATONIN AND THE CIRCADIAN MODULATION OF MEMORY PROCESSES IN ZEBRAFISH}

Zebrafish (Danio rerio), a diurnal organism, was used as a model system to investigate the role of the circadian system in modulating learning and memory formation, using a modified active-avoidance conditioning (AAC) paradigm (Rawashdeh et al., 2007). Animals were placed within a tank of two equal sized compartments. Both compartments were separated by a semi-partition allowing the fish to swim freely between both compartments. In this task, animals had to learn to associate a compartment within the training tank having a light signal (conditioned stimulus-CS) as the safe environment and/or the dark compartment associated with mild electric shocks (unconditioned stimulus-US) as the unsafe environment.

When fish were trained during their active phase (daytime) under a light/dark cycle, they demonstrated a shortening in the time required to meet the learning criteria as compared to training during the nighttime. Furthermore, when retraining animals $24 \mathrm{~h}$ later, to measure for memory formation, only those animals trained during the light period showed improved performance when compared to the initial training session. This diurnal pattern in the rate of acquisition persisted also under constant conditions (darkness), however, with a clear difference in the amplitude of the rhythm. More precisely, the performance in the rate of relearning when trained $24 \mathrm{~h}$ later during the subjective daytime was significantly reduced as compared to animals trained at the same phase but under light/dark conditions. It was hypothesized then, that in the presence of light the additional reduction of daytime melatonin levels in zebrafish, further decreases a melatonin mediated inhibition on memory consolidation. To test this hypothesis, the action of pharmacologically applied melatonin was tested on memory performance during the subjective daytime when memory formation for AAC was enhanced. From the dose response curve for melatonin action on memory performance it was demonstrated that melatonin has indeed an inhibitory effect on memory consolidation.

Importantly, melatonin was found to be effective in interfering with long-term memory formation for AAC, however, only when it was applied prior to training. Furthermore, no effect of melatonin on memory retrieval was observed when animals were given melatonin prior to testing the animal's performance after $24 \mathrm{~h}$. The later suggests that melatonin does not obstruct the retrieval of the newly formed memory for AAC. In conclusion, melatonin has a profound influence on an early yet 
necessary event required for memory consolidation in zebrafish. If endogenous nighttime melatonin had an effect on memory retrieval it would have become apparent when assaying AAC performance for daytime trained animals during nighttime, $36 \mathrm{~h}$ instead of $24 \mathrm{~h}$ later. This, however, was not the case, since the time of training not the time of testing was the determining factor in the animal's performance. Meaning, memory consolidation not retrieval is modulated by the circadian system.

The specificity of the effect of pharmacological melatonin treatment on memory consolidation to be mediated by its receptors was confirmed using $\mathrm{MT}(1) / \mathrm{MT}(2)$ receptor antagonists prior to the melatonin treatment. It was next hypothesized that by blocking nighttime melatonin signaling using only melatonin receptor antagonists, that nighttime impairments in memory consolidation for AAC could be lifted. Indeed, training zebrafish during the nighttime, when memory consolidation following nighttime acquisition is weakest, mimicked daytime performance for AAC.

To confirm that endogenous melatonin plays a role in the circadian modulation of memory performance, demanded the removal of the main source of melatonin synthesis, namely the pineal gland (Figure 1). The data show that pinealectomy per se has no effect on acquisition and on the improvement of daytime AAC performance during testing as compared to sham operated animals. In contrast to daytime memory performance for AAC, nighttime memory performance was different. During nighttime, when pinealectomized animals were trained for AAC, zebrafish demonstrate memory performance for AAC similar to their performances for normal daytime trained animals. Therefore, it was concluded that endogenous nighttime melatonin has an inhibitory effect on zebrafish nighttime AAC performance. Whether this effect of melatonin is species specific and/or dependent on the activity profile of the animal model (diurnal vs. nocturnal) remains to be investigated.

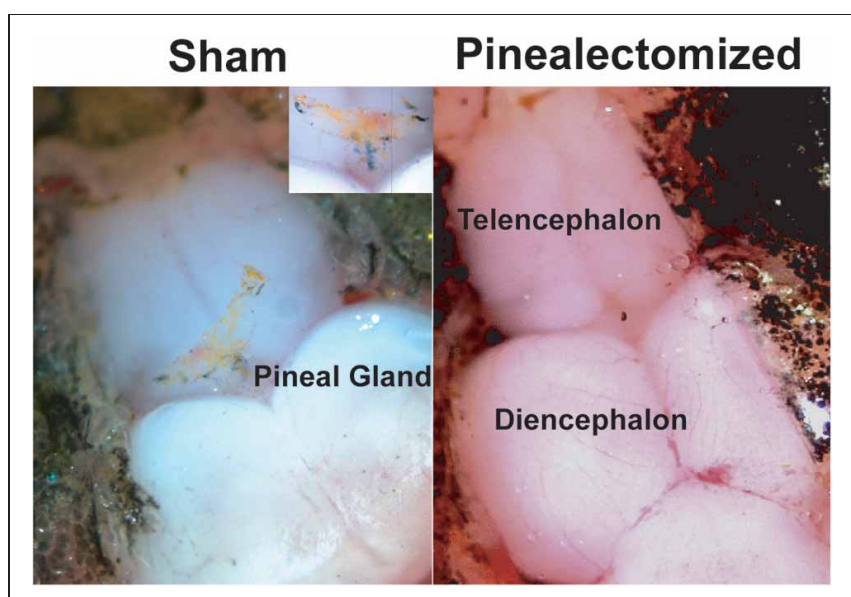

FIGURE 1 | Representative pictures of zebrafish brain. Left: Sham operated animal depicting an intact pineal gland (see also inset).

Right: Photograph of pinealectomized zebrafish brain. Note the absence of a pineal gland between the telencephalon and the diencephalon in the photograph to the right as compared to the left.
However, in the case of zebrafish, we conclude that cycling physiological melatonin is one mechanism by which the circadian system is rhythmically modulating memory processing for AAC (Figure 2).

\section{FROM ZEBRAFISH TO HUMAN}

The findings that nighttime melatonin may actively suppress memory consolidation following nighttime acquisition may implicate a similar endogenous role for melatonin in humans. Note that under normal (healthy) physiological conditions the direct effect of melatonin on human cognition has not been investigated to the best of our knowledge. The only data available suggesting a link between endogenous melatonin rhythm and cognition in humans comes from studies in which the population sample chosen falls within a specific category of disease (Beversdorf et al., 2000) or age (Touitou et al., 1981; Savaskan et al., 2005; Brunner et al., 2006), concurrent to abnormalities in melatonin rhythms in humans. Although several studies included in their investigation, established questionnaires to evaluate the impact of melatonin on mental performance, this area of research remains at its infancy. While, we do not expect nighttime melatonin to be detrimental for proper memory consolidation for tasks acquired during daytime, we hypothesize that nighttime melatonin does have an effect on memories to be formed for tasks learned during nighttime. This, however, will be a very

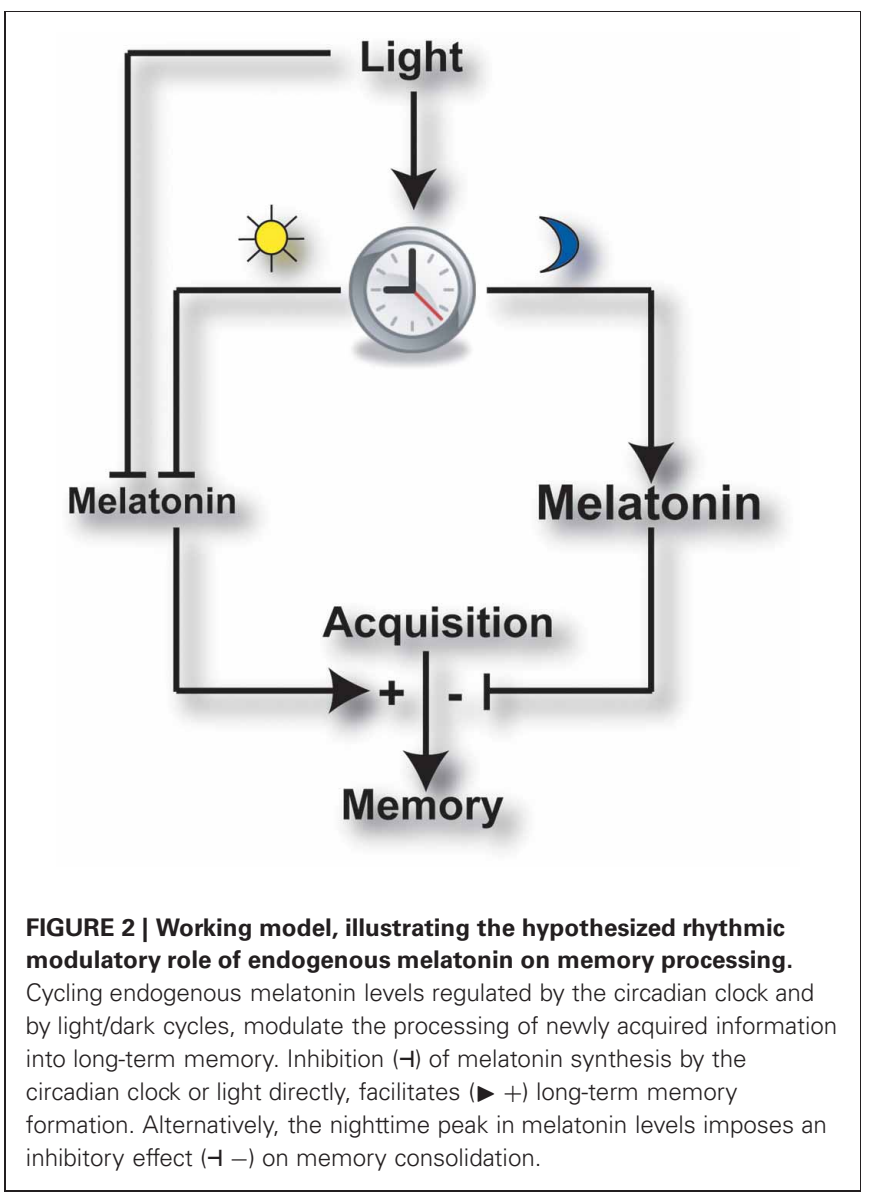


challenging task, as it calls to dissociate the impact of sleep-loss on the expense of nighttime learning from that of the hypothesized effect of melatonin on memory for newly nighttime acquired tasks.

Nevertheless, the improvement of memory by blocking nocturnal melatonin signaling in zebrafish, has some symptomatic resemblance to autism spectrum disorder individuals showing high memory capacities (Beversdorf et al., 2000) associated with abnormally low and/or arrhythmic $24 \mathrm{~h}$ melatonin levels (Melke et al., 2008). In these individuals, applied melatonin treatments demonstrated to be beneficial in several of the symptoms associated with autism. However, since enhanced memory capacity in these individuals is secondary at least in the eyes of the parents

\section{REFERENCES}

Aeschbach, D., Lockyer, B. J., Dijk, D. J., Lockley, S. W., Nuwayser, E. S., Nichols, L. D., and Czeisler, C. A. (2009). Use of transdermal melatonin delivery to improve sleep maintenance during daytime. Clin. Pharmacol. Ther. 86, 378-382.

Antoniadis, E. A., Ko, C. H., Ralph, M. R., and McDonald, R. J. (2000). Circadian rhythms, aging and memory. Behav. Brain Res. 111, 25-37.

Arendt, J., and Broadway, J. (1987). Light and melatonin as Zeitgebers in man. Chronobiol. Int. 4, 273-282.

Armstrong, S. M., Cassone, V. M., Chesworth, M. J., Redman, J. R., and Short, R. V. (1986). Synchronization of mammalian circadian rhythms by melatonin. J. Neural Transm. Suppl. 21, 375-394.

Bartell, P. A., Miranda-Anaya, M., and Menaker, M. (2004). Period and phase control in a multioscillatory circadian system (Iguana iguana). J. Biol. Rhythms 19, 47-57.

Baskett, J. J., Wood, P. C., Broad, J. B., Duncan, J. R., English, J., and Arendt, J. (2001). Melatonin in older people with age-related sleep maintenance problems: a comparison with age matched normal sleepers. Sleep 24, 418-424.

Beversdorf, D. Q., Smith, B. W., Crucian, G. P., Anderson, J. M., Keillor, J. M., Barrett, A. M., Hughes, J. D., Felopulos, G. J., Bauman, M. L., Nadeau, S. E., and Heilman, K. M. (2000). Increased discrimination of "false memories" in autism spectrum disorder. Proc. Natl. Acad. Sci. U.S.A. 97, 8734-8737.

Binkley, S., Kluth, E., and Menaker, M. (1971). Pineal function in sparrows: circadian rhythms and body temperature. Science 174, 311-314.

Bob, P., and Fedor-Freybergh, P. (2008). Melatonin, consciousness, and traumatic stress. J. Pineal Res. 44, 341-347.
Brunner, P., Sozer-Topcular, N., Jockers, R., Ravid, R., Angeloni, D., Spahn, F., and Savaskan, E. (2006). Pineal and cortical melatonin receptors MT1 and MT2 are decreased in Alzheimer's disease. Eur. J. Histochem. 50, 311-316.

Cahill, G. M. (1996). Circadian regucultured zebrafish pineal and retina. Brain Res. 708, 177-181.

Cahill, G. M., Hurd, M. W., and Batchelor, M. M. (1998). Circadian rhythmicity in the locomotor activity of larval zebrafish. Neuroreport 9, 3445-3449.

Cassone, V. M. (1990). Effects of melatonin on vertebrate circadian systems. Trends Neurosci. 13, 457-464.

Castillo, M. R., Hochstetler, K. J., Greene, D. M., Firmin, S. I., Tavernier, R. J., Raap, D. K., and Bult-Ito, A. (2005). Circadian rhythm of core body temperature in two laboratory mouse lines. Physiol. Behav. 86, 538-545.

Chaudhury, D., and Colwell, C. S. (2002). Circadian modulation of learning and memory in fearconditioned mice. Behav. Brain Res. 133, 95-108.

Cheung, P. W., and McCormack, C. E. (1982). Failure of pinealectomy or melatonin to alter circadian activity rhythm of the rat. Am. J. Physiol. 242, R261-R264.

Collins, D. R., and Davies, S. N. (1997). Melatonin blocks the induction of long-term potentiation in an $\mathrm{N}$-methyl-D-aspartate independent manner. Brain Res. 767, 162-165.

Devan, B. D., Goad, E. H., Petri, H. L., Antoniadis, E. A., Hong, N. S., Ko, C. H., Leblanc, L., Lebovic, S. S., Lo, Q., Ralph, M. R., and McDonald, R. J. (2001). Circadian phase-shifted rats show normal acquisition but impaired long-term retention of place information in the water task. Neurobiol. Learn. Mem. 75, 51-62. Fraschini, F., Eckert, A., Mullerlation of melatonin production in

as compared to severe social retrieval and sleep disturbances, the effect of such melatonin treatments on their mental performance has not yet been investigated.

Importantly, the finding that by blocking melatonin signaling, nighttime acquisition is translated into a long-term memory encourages further research as a possible therapeutic or perhaps as a short-term cognitive compensatory treatment for people subjected to abnormal sleep/wake cycles to improve temporary deficits in mental performance.

\section{ACKNOWLEDGMENTS}

We thank Prof. Dr. Jörg H. Stehle for his input, discussions, and critical reading of this manuscript.

Dubocovich, M. L. (1988). Luzindole (N-0774): a novel melatonin receptor antagonist. J. Pharmacol. Exp. Ther. 246, 902-910.

Fekete, M., Van Ree, J. M., Niesink, R. J. M., and De Wied, D. (1985). Disrupting circadian rhythms in rats induces retrograde amnesia. Physiol. Behav. 34, 883-887.

Fernandez, R. I., Lyons, L. C., Levenson, J., Khabour, O., and Eskin, A. (2003). Circadian modulation of long-term sensitization in Aplysia. Proc. Natl. Acad. Sci. U.S.A. 100 14415-14420.

Fisher, S. P., and Sugden, D. (2010). Endogenous melatonin is not obligatory for the regulation of the rat sleep-wake cycle. Sleep 33 , 833-840.

Gastel, J. A., Roseboom, P. H., Rinaldi, P. A., Weller, J. L., and Klein, D. C. (1998). Melatonin production: proteasomal proteolysis in serotonin $\mathrm{N}$-acetyltransferase regulation. Science 279, 1358-1360.

Gaston, S., and Menaker, M. (1968). Pineal function: the biological clock in the sparrow? Science 160 1125-1127.

Gerstner, J. R., Lyons, L. C., Wright, K. P. Jr., Loh, D. H., Rawashdeh, O., Eckel-Mahan, K. L., and Roman, G. W. (2009). Cycling behavior and memory formation. J. Neurosci. 29 12824-12830.

Hogan, M. V., El-Sherif, Y., and Wieraszko, A. (2001). The modulation of neuronal activity by melatonin: in vitro studies on mouse hippocampal slices. J. Pineal Res. 30, 87-96.

Hughes, R. J., Sack, R. L., and Lewy, A. J. (1998). The role of melatonin and circadian phase in agerelated sleep-maintenance insomnia: assessment in a clinical trial of melatonin replacement. Sleep 21, 52-68.

Hunt, A. E., Al-Ghoul, W. M., Gillette, M. U., and Dubocovich, M. L.
(2001). Activation of MT(2) melatonin receptors in rat suprachiasmatic nucleus phase advances the circadian clock. Am. J. Physiol. Cell Physiol. 280, C110-C118.

Hurd, M. W., Debruyne, J., Straume, M., and Cahill, G. M. (1998). Circadian rhythms of locomotor activity in zebrafish. Physiol. Behav. 65, 465-472.

Kaneko, M., Hernandez-Borsetti, N. and Cahill, G. M. (2006). Diversity of zebrafish peripheral oscillators revealed by luciferase reporting. Proc. Natl. Acad. Sci. U.S.A. 103 14614-14619.

Klein, D. C., Coon, S. L., Roseboom, P. H., Weller, J. L., Bernard, M., Gastel, J. A., Zatz, M., Iuvone, P. M., Rodriguez, I. R., Begay, V., Falcon, J., Cahill, G. M., Cassone, V. M., and Baler, R. (1997). The melatonin rhythm-generating enzyme: molecular regulation of serotonin $\mathrm{N}$ acetyltransferase in the pineal gland. Recent Prog. Horm. Res. 52, 307-357; discussion 357-308.

Korf, H. W., Schomerus, C., and Stehle, J. H. (1998). The pineal organ, its hormone melatonin, and the photoneuroendocrine system. Adv. Anat. Embryol. Cell Biol. 146, 1-100.

Kroeber, S., Meissl, H., Maronde, E., and Korf, H. W. (2000). Analyses of signal transduction cascades reveal an essential role of calcium ions for regulation of melatonin biosynthesis in the light-sensitive pineal organ of the rainbow trout (Oncorhynchus mykiss). J. Neurochem. 74, 2478-2489.

Larson, J., Jessen, R. E., Uz, T., Arslan, A. D., Kurtuncu, M., Imbesi, M., and Manev, H. (2006). Impaired hippocampal long-term potentiation in melatonin MT2 receptordeficient mice. Neurosci. Lett. 393, 23-26.

Lavie, P. (1997). Melatonin: role in gating nocturnal rise in sleep propensity. J. Biol. Rhythms 12, 657-665. 
Lerner, A. B., Case, J. D., Takahashi, Y., Lee, T. H., and Mori, W. (1958). Isolation of melatonin, the pineal gland factor that lightens melanocyteS1. J. Am. Chem. Soc. 80, 2587-2587.

Lyons, L. C., Rawashdeh, O., and Eskin, A. (2006). Non-ocular circadian oscillators and photoreceptors modulate long term memory formation in Aplysia. J. Biol. Rhythms 21, 245-255

Lyons, L. C., Rawashdeh, O., Katzoff, A., Susswein, A. J., and Eskin, A. (2005). Circadian modulation of complex learning in diurnal and nocturnal Aplysia. Proc. Natl. Acad. Sci. U.S.A. 102, 12589-12594.

McArthur, A. J., Hunt, A. E., and Gillette, M. U. (1997). Melatonin action and signal transduction in the rat suprachiasmatic circadian clock: activation of protein kinase $\mathrm{C}$ at dusk and dawn. Endocrinology 138, 627-634.

Melke, J., Goubran Botros, H., Chaste, P., Betancur, C., Nygren, G., Anckarsater, H., Rastam, M., Stahlberg, O., Gillberg, I. C., Delorme, R., Chabane, N., MourenSimeoni, M. C., Fauchereau, F., Durand, C. M., Chevalier, F., Drouot, X., Collet, C., Launay, J. M., Leboyer, M., Gillberg, C., and Bourgeron, T. (2008). Abnormal melatonin synthesis in autism spectrum disorders. Mol. Psychiatry 13, 90-98.
Petrie, K., Conaglen, J. V., Thompson, L., and Chamberlain, K. (1989). Effect of melatonin on jet lag after long haul flights. BMJ 298, 705-707.

Pozdeyev, N., Taylor, C., Haque, R., Chaurasia, S. S., Visser, A. Thazyeen, A., Du, Y., Fu, H., Weller, J., Klein, D. C., and Iuvone, P. M. (2006). Photic regulation of arylalkylamine $\mathrm{N}$-acetyltransferase binding to 14-3-3 proteins in retinal photoreceptor cells. J. Neurosci. 26, 9153-9161.

Rawashdeh, O., De Borsetti, N. H., Roman, G., and Cahill, G. M. (2007). Melatonin suppresses nighttime memory formation in zebrafish. Science 318, 1144-1146.

Reppert, S. M., Godson, C., Mahle, C. D., Weaver, D. R., Slaugenhaupt, S. A., and Gusella, J. F. (1995). Molecular characterization of a second melatonin receptor expressed in human retina and brain: the Mellb melatonin receptor. Proc. Natl. Acad. Sci. U.S.A. 92, 8734-8738.

Roberson, E. D., and Sweatt, J. D. (1996). Transient activation of cyclic AMP-dependent protein kinase during hippocampal longterm potentiation. J. Biol. Chem. 271, 30436-30441.

Sack, R. L., Lewy, A. J., and Hughes, R. J. (1998). Use of melatonin for sleep and circadian rhythm disorders. Ann. Med. 30, 115-121.
Savaskan, E., Ayoub, M. A., Ravid, R. Angeloni, D., Fraschini, F., Meier, F., Eckert, A., Muller-Spahn, F., and Jockers, R. (2005). Reduced hippocampal MT2 melatonin receptor expression in Alzheimer's disease. J. Pineal Res. 38, 10-16.

Stokkan, K. A., Yamazaki, S., Tei, H. Sakaki, Y., and Menaker, M. (2001). Entrainment of the circadian clock in the liver by feeding. Science 291, 490-493.

Takahashi, J. S., and Zatz, M. (1982). Regulation of circadian rhythmicity. Science 217, 1104-1111.

Tapp, W. N., and Holloway, F. A. (1981). Phase shifting circadian rhythms produces retrograde amnesia. Science 211, 1056-1058.

Touitou, Y., Fevre, M., Lagoguey, M., Carayon, A., Bogdan, A., Reinberg, A., Beck, H., Cesselin, F., and Touitou, C. (1981). Age- and mental health-related circadian rhythms of plasma levels of melatonin, prolactin, luteinizing hormone and follicle-stimulating hormone in man. J. Endocrinol. 91, 467-475.

Turek, F. W., McMillan, J. P., and Menaker, M. (1976). Melatonin: effects on the circadian locomotor rhythm of sparrows. Science 194 1441-1443.

Underwood, H. (1983). Circadian pacemakers in lizards: phaseresponse curves and effects of pinealectomy. Am. J. Physiol. 244, R857-R864.
Wang, L. M., Suthana, N. A., Chaudhury, D., Weaver, D. R., and Colwell, C. S. (2005). Melatonin inhibits hippocampal long-term potentiation. Eur. J. Neurosci. 22, 2231-2237.

Zimmerman, N. H., and Menaker, M. (1975). Neural connections of sparrow pineal: role in circadian control of activity. Science 190, 477-479.

Conflict of Interest Statement: The authors declare that the research was conducted in the absence of any commercial or financial relationships that could be construed as a potential conflict of interest.

Received: 02 January 2012; paper pending published: 03 February 2012; accepted: 15 February 2012; published online: 06 March 2012.

Citation: Rawashdeh $O$ and Maronde E (2012) The hormonal Zeitgeber melatonin: role as a circadian modulator in memory processing. Front. Mol. Neurosci. 5:27. doi: 10.3389/fnmol. 2012.00027

Copyright (c) 2012 Rawashdeh and Maronde. This is an open-access article distributed under the terms of the Creative Commons Attribution Non Commercial License, which permits noncommercial use, distribution, and reproduction in other forums, provided the original authors and source are credited. 